\title{
Boundedness for commutators of fractional $p$-adic Hardy operators
}

\section{Qing Yan $W u^{*}$}

\section{"Correspondence:}

qingyanwu@lyu.edu.cn

School of Science, Linyi University,

Linyi, 276005, P.R. China

\begin{abstract}
In this paper we prove that the commutators generated by the fractional $p$-adic Hardy operators and the central BMO function are bounded on weighted homogeneous Herz spaces.

MSC: 11E95; 11K70; 42B99

Keywords: fractional $p$-adic Hardy operator; central BMO function; weighted homogeneous Herz space
\end{abstract}

\section{Introduction}

In recent years, $p$-adic fields have been introduced into some aspects of mathematical physics. There are a lot of articles where different applications of the $p$-adic analysis in the string theory, quantum mechanics, stochastics, the theory of dynamical systems, cognitive sciences, and psychology are studied [1-9] (see also the references therein). As a consequence, new mathematical problems have emerged, among them, the study of harmonic analysis on a $p$-adic field has been drawing more and more concern ( $c f$. [10-14] and the references therein).

For a prime number $p$, let $\mathbb{Q}_{p}^{n}$ be the field of $p$-adic numbers. It is defined as the completion of the field of rational numbers $\mathbb{Q}$ with respect to the non-Archimedean $p$-adic norm $|\cdot|_{p}$. This norm is defined as follows: $|0|_{p}=0$. If any non-zero rational number $x$ is represented as $x=p^{\gamma} \frac{m}{n}$, where $m$ and $n$ are integers which are not divisible by $p$, and $\gamma$ is an integer, then $|x|_{p}=p^{-\gamma}$. It is not difficult to show that the norm satisfies the following properties:

$$
|x y|_{p}=|x|_{p}|y|_{p}, \quad|x+y|_{p} \leq \max \left\{|x|_{p},|y|_{p}\right\} .
$$

It follows from the second property that when $|x|_{p} \neq|y|_{p}$, then $|x+y|_{p}=\max \left\{|x|_{p},|y|_{p}\right\}$. From the standard $p$-adic analysis [7], we see that any non-zero $p$-adic number $x \in \mathbb{Q}_{p}$ can be uniquely represented in the canonical series

$$
x=p^{\gamma} \sum_{j=0}^{\infty} a_{j} p^{j}, \quad \gamma=\gamma(x) \in \mathbb{Z},
$$

where $a_{j}$ are integers, $0 \leq a_{j} \leq p-1, a_{0} \neq 0$. The series (1.1) converges in the $p$-adic norm because $\left|a_{j} p^{j}\right|_{p}=p^{-j}$. Set $\mathbb{Q}_{p}^{*}=\mathbb{Q}_{p} \backslash\{0\}$.

\section{Springer}

(0 2012 Wu; licensee Springer. This is an Open Access article distributed under the terms of the Creative Commons Attribution License (http://creativecommons.org/licenses/by/2.0), which permits unrestricted use, distribution, and reproduction in any medium, provided the original work is properly cited. 
The space $\mathbb{Q}_{p}^{n}$ consists of points $x=\left(x_{1}, x_{2}, \ldots, x_{n}\right)$, where $x_{j} \in \mathbb{Q}_{p}, j=1,2, \ldots, n$. The $p$ adic norm on $\mathbb{Q}_{p}^{n}$ is

$$
|x|_{p}:=\max _{1 \leq j \leq n}\left|x_{j}\right|_{p}, \quad x \in \mathbb{Q}_{p}^{n} .
$$

Denote by

$$
B_{\gamma}(a)=\left\{x \in \mathbb{Q}_{p}^{n}:|x-a|_{p} \leq p^{\gamma}\right\},
$$

the ball with center at $a \in \mathbb{Q}_{p}^{n}$ and radius $p^{\gamma}$, and

$$
S_{\gamma}(a)=\left\{x \in \mathbb{Q}_{p}^{n}:|x-a|_{p}=p^{\gamma}\right\}=B_{\gamma}(a) \backslash B_{\gamma-1}(a) .
$$

Since $\mathbb{Q}_{p}^{n}$ is a locally compact commutative group under addition, it follows from the standard analysis that there exists a Haar measure $d x$ on $\mathbb{Q}_{p}^{n}$, which is unique up to a positive constant multiple and is translation invariant. We normalize the measure $d x$ by the equality

$$
\int_{B_{0}(0)} d x=\left|B_{0}(0)\right|_{H}=1,
$$

where $|E|_{H}$ denotes the Haar measure of a measurable subset $E$ of $\mathbb{Q}_{p}^{n}$. By simple calculation, we can obtain that

$$
\left|B_{\gamma}(a)\right|_{H}=p^{\gamma n}, \quad\left|S_{\gamma}(a)\right|_{H}=p^{\gamma n}\left(1-p^{-n}\right),
$$

for any $a \in \mathbb{Q}_{p}^{n}$. For a more complete introduction to the $p$-adic field, see [15] or [7].

The classical Hardy operators are defined by

$$
H f(x):=\frac{1}{x} \int_{0}^{x} f(t) d t, \quad H^{*} f(x):=\int_{x}^{\infty} \frac{f(t)}{t} d t, \quad x>0,
$$

for a non-negative integrable function $f$ on $\mathbb{R}^{+}$. Obviously, $\mathcal{H}$ and $\mathcal{H}^{*}$ satisfy

$$
\int_{\mathbb{R}^{n}} g(x) \mathcal{H} f(x) d x=\int_{\mathbb{R}^{n}} f(x) \mathcal{H}^{\prime \prime} g(x) d x .
$$

The well-known Hardy integral inequality [16] tells us that for $1<q<\infty$,

$$
\|H f\|_{L^{q}\left(\mathbb{R}^{+}\right)} \leq \frac{q}{q-1}\|f\|_{L^{q\left(\mathbb{R}^{+}\right)}}
$$

where the constant $\frac{q}{q-1}$ is the best possible. The generalized result [17] is that

$$
\left\|H^{\prime \prime} f\right\|_{L^{q^{\prime}\left(\mathbb{R}^{+}\right)}} \leq \frac{q}{q-1}\|f\|_{L^{q^{\prime}\left(\mathbb{R}^{+}\right)}},
$$

and

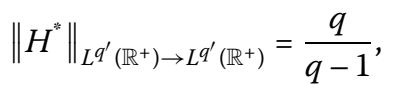

where $\frac{1}{q}+\frac{1}{q^{\prime}}=1$. 
The Hardy integral inequalities have received considerable attention due to their usefulness in analysis and their applications. There are numerous papers dealing with their various generalizations, variants and applications ( $c f$. [18-20] and the references cited therein). We have obtained the Hardy integral inequalities for $p$-adic Hardy operators and their commutators [21]. The boundedness of commutators is an active topic in harmonic analysis because of its important applications; for example, it can be applied to characterizing some function spaces. There are a lot of works about the boundedness of commutators of various Hardy-type operators on Euclidean spaces (cf. [22, 23], etc.). In this paper, we will establish the Hardy integral inequalities for commutators generated by fractional $p$-adic Hardy operators and CMO functions.

Definition 1.1 For a function $f$ on $\mathbb{Q}_{p}^{n}$, we define the $p$-adic Hardy operators as follows:

$$
\begin{aligned}
& \mathcal{H}^{p} f(x)=\frac{1}{|x|_{p}^{n}} \int_{B\left(0,|x|_{p}\right)} f(t) d t, \\
& \mathcal{H}^{p, *} f(x)=\int_{\mathbb{Q}_{p}^{n} \backslash B(0,|x| p)} \frac{f(t)}{|t|_{p}^{n}} d t, \quad x \in \mathbb{Q}_{p}^{n} \backslash\{0\},
\end{aligned}
$$

where $B\left(0,|x|_{p}\right)$ is a ball in $\mathbb{Q}_{p}^{n}$ with center at $0 \in \mathbb{Q}_{p}^{n}$ and radius $|x|_{p}$.

Definition 1.2 Let $f \in L_{\mathrm{loc}}\left(\mathbb{Q}_{p}^{n}\right), 0 \leq \beta<n$. The fractional $p$-adic Hardy operators are defined by

$$
\begin{aligned}
& \mathcal{H}_{\beta}^{p} f(x)=\frac{1}{|x|_{p}^{n-\beta}} \int_{B(0,|x| p)} f(t) d t, \\
& \mathcal{H}_{\beta}^{p, p^{*}} f(x)=\int_{\mathbb{Q}_{p}^{n} \backslash B\left(0,|x|_{p}\right)} \frac{f(t)}{|t|_{p}^{n-\beta}} d t, \quad x \in \mathbb{Q}_{p}^{n} \backslash\{0\},
\end{aligned}
$$

where $B\left(0,|x|_{p}\right)$ is the ball as in Definition 1.1.

It is clear that when $\beta=0$, then $\mathcal{H}_{\beta}^{p}$ becomes $\mathcal{H}^{p}$.

Definition 1.3 Let $b \in L_{\mathrm{loc}}\left(\mathbb{Q}_{p}^{n}\right), 0 \leq \beta<n$. The commutators of fractional $p$-adic Hardy operators are defined by

$$
\mathcal{H}_{\beta, b}^{p} f=b \mathcal{H}_{\beta}^{p} f-\mathcal{H}_{\beta}^{p}(b f), \quad \mathcal{H}_{\beta, b}^{p,{ }^{*}} f=b \mathcal{H}_{\beta}^{p,{ }^{, *}} f-\mathcal{H}_{\beta}^{p,{ }^{*}}(b f) .
$$

In [24-26], the CMO spaces (central BMO spaces) on $\mathbb{R}^{n}$ have been introduced and studied. CMO spaces bear a simple relationship with BMO: $g \in B M O$ precisely when $g$ and all of its translates belong to BMO spaces uniformly a.e. Many precise analogies exist between $\mathrm{CMO}$ spaces and $\mathrm{BMO}$ spaces from the point of view of real Hardy spaces. Similarly, we define the $C M O^{q}$ spaces on $\mathbb{Q}_{p}^{n}$.

Definition 1.4 Let $1 \leq q<\infty$, a function $f \in L_{\text {loc }}^{q}\left(\mathbb{Q}_{p}^{n}\right)$ is said to be in $C M O^{q}\left(\mathbb{Q}_{p}^{n}\right)$ if

$$
\|f\|_{C M O^{q}\left(\mathbb{Q}_{p}^{n}\right)}:=\sup _{\gamma \in \mathbb{Z}}\left(\frac{1}{\left|B_{\gamma}(0)\right|_{H}} \int_{B_{\gamma(0)}}\left|f(x)-f_{B_{\gamma(0)}}\right|^{q} d x\right)^{\frac{1}{q}}<\infty,
$$


where

$$
f_{B_{\gamma(0)}}=\frac{1}{\left|B_{\gamma}(0)\right|_{H}} \int_{B_{\gamma(0)}} f(x) d x .
$$

Remark 1.1 It is obvious that $L^{\infty}\left(\mathbb{Q}_{p}^{n}\right) \subset B M O\left(\mathbb{Q}_{p}^{n}\right) \subset C M O^{q}\left(\mathbb{Q}_{p}^{n}\right)$.

Let $B_{k}=B_{k}(0)=\left\{x \in \mathbb{Q}_{p}^{n}:|x|_{p} \leq p^{k}\right\}, S_{k}=B_{k} \backslash B_{k-1}$ and $\chi_{k}$ be the characteristic function of the set $S_{k}$.

Definition 1.5 [27] Suppose that $\alpha \in \mathbb{R}, 0<q<\infty$ and $0<r<\infty$. The homogeneous $p$-adic Herz space $K_{r}^{\alpha, q}\left(\mathbb{Q}_{p}^{n}\right)$ is defined by

$$
K_{r}^{\alpha, q}\left(\mathbb{Q}_{p}^{n}\right)=\left\{f \in L_{\mathrm{loc}}^{r}\left(\mathbb{Q}_{p}^{n}\right):\|f\|_{K_{r}^{\alpha, q}\left(\mathbb{Q}_{p}^{n}\right)}<\infty\right\},
$$

where

$$
\|f\|_{K_{r}^{\alpha, q}\left(\mathbb{Q}_{p}^{n}\right)}=\left(\sum_{k=-\infty}^{+\infty} p^{k \alpha q}\left\|f \chi_{k}\right\|_{L^{r}\left(\mathbb{Q}_{p}^{n}\right)}^{q}\right)^{\frac{1}{q}}
$$

with the usual modifications made when $q=\infty$ or $r=\infty$.

Remark 1.2 $K_{r}^{\alpha, q}\left(\mathbb{Q}_{p}^{n}\right)$ is the generalization of $L^{q}\left(|x|_{p}^{\alpha} d x\right)$, and $K_{q}^{0, q}\left(\mathbb{Q}_{p}^{n}\right)=L^{q}\left(\mathbb{Q}_{p}^{n}\right)$, $K_{q}^{\frac{\alpha}{q}, q}\left(\mathbb{Q}_{p}^{n}\right)=L^{q}\left(|x|_{p}^{\alpha} d x\right)$ for all $0<q \leq \infty$ and $\alpha \in \mathbb{R}$.

Motivated by [22], we get the following operator boundedness results. Throughout this paper, we use $C$ to denote different positive constants which are independent of the essential variables.

Theorem 1.1 Suppose that $\beta \geq 0,0<q_{1} \leq q_{2}<\infty, \frac{1}{r_{1}}-\frac{1}{r_{2}}=\frac{\beta}{n}, 1<r_{1}<\infty, \frac{1}{r_{1}}+\frac{1}{r_{1}^{\prime}}=1$, $b \in C M O^{\max \left\{r_{1}^{\prime}, r_{2}\right\}}\left(\mathbb{Q}_{p}^{n}\right)$. Then

(1) If $\alpha<\frac{n}{r_{1}^{\prime}}$, then

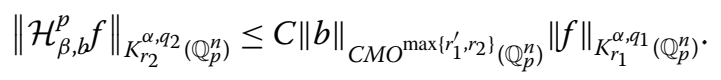

(2) If $\alpha>-\frac{n}{r_{2}}$, then

$$
\left\|\mathcal{H}_{\beta, b}^{p, *} f\right\|_{K_{r_{2}}^{\alpha, q_{2}}\left(\mathbb{Q}_{p}^{n}\right)} \leq C\|b\|_{C M O^{\max \left(r_{1}^{\prime}, r_{2}\right\}}\left(\mathbb{Q}_{p}^{n}\right)}\|f\|_{K_{r_{1}}^{\alpha, q_{1}}\left(\mathbb{Q}_{p}^{n}\right)}
$$

When $\alpha=0, q_{j}=r_{j}, j=1,2$, we can get the following result.

Corollary 1.1 Suppose that $\beta \geq 0,0<q_{1} \leq q_{2}<\infty, \frac{1}{q_{1}}-\frac{1}{q_{2}}=\frac{\beta}{n}, 1<q_{1}<\infty, \frac{1}{q_{1}}+\frac{1}{q_{1}^{\prime}}=1$, $b \in C M O^{\max \left\{q_{1}^{\prime}, q_{2}\right\}}\left(\mathbb{Q}_{p}^{n}\right)$. Then

$$
\left\|\mathcal{H}_{\beta, b}^{p} f\right\|_{L^{q_{2}\left(\mathbb{Q}_{p}^{n}\right)}} \leq C\|b\|_{C M O^{\max \left\{q_{1}^{\prime}, q_{2}\right\}}\left(\mathbb{Q}_{p}^{n}\right)}\|f\|_{L^{q_{1}}\left(\mathbb{Q}_{p}^{n}\right)},
$$


and

$$
\left\|\mathcal{H}_{\beta, b}^{p, *} f\right\|_{L^{q_{2}\left(\mathbb{Q}_{p}^{n}\right)}} \leq C\|b\|_{C M O^{\max \left\{q_{1}^{\prime}, q_{2}\right\}}\left(\mathbb{Q}_{p}^{n}\right)}\|f\|_{L^{q_{1}\left(\mathbb{Q}_{p}^{n}\right)}}
$$

When $\beta=0$, we can get the boundedness of a $p$-adic Hardy operator in [21].

Corollary 1.2 Let $0<q_{1} \leq q_{2}<\infty, 1<r<\infty, b \in C M O^{\max \left\{r, r^{\prime}\right\}}\left(\mathbb{Q}_{p}^{n}\right)$. Then

(1) If $\alpha<\frac{n}{r^{\prime}}$, then

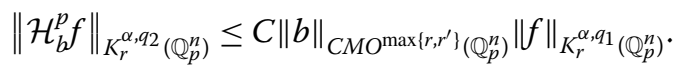

(2) If $\alpha>-\frac{n}{r}$, then

$$
\left\|\mathcal{H}_{b}^{p, *} f\right\|_{K_{r_{2}}^{\alpha, q_{2}}\left(\mathbb{Q}_{p}^{n}\right)} \leq C\|b\|_{C M O^{\max \left\{r, r^{\prime}\right\}}\left(\mathbb{Q}_{p}^{n}\right)}\|f\|_{K_{r_{1}}^{\alpha, q_{1}}\left(\mathbb{Q}_{p}^{n}\right)} .
$$

By the similar proof of Theorem 1.1, we can obtain the following result.

Corollary 1.3 Suppose that $\beta \geq 0,0<q_{1} \leq q_{2}<\infty, \frac{1}{r_{1}}-\frac{1}{r_{2}}=\frac{\beta}{n}, 1<r_{1}<\infty, \frac{1}{r_{1}}+\frac{1}{r_{1}^{\prime}}=1$. Then

(1) If $\alpha<\frac{n}{r_{1}^{\prime}}$, then

$$
\left\|\mathcal{H}_{\beta}^{p} f\right\|_{K_{r_{2}}^{\alpha, q_{2}}\left(\mathbb{Q}_{p}^{n}\right)} \leq C\|f\|_{K_{r_{1}}^{\alpha, q_{1}}\left(\mathbb{Q}_{p}^{n}\right)} \cdot
$$

(2) If $\alpha>-\frac{n}{r_{2}}$, then

$$
\left\|\mathcal{H}_{\beta}^{p, *} f\right\|_{K_{r_{2}}^{\alpha, q_{2}}\left(\mathbb{Q}_{p}^{n}\right)} \leq C\|f\|_{K_{r_{1}}^{\alpha, q_{1}}\left(\mathbb{Q}_{p}^{n}\right)} .
$$

\section{Boundedness of commutators of fractional $p$-adic Hardy operator} In order to prove Theorem 1.1, we firstly give the following lemmas.

Lemma 2.1 Suppose that $b$ is a CMO function and $1 \leq q<r<\infty$, then $C M O^{r}\left(\mathbb{Q}_{p}^{n}\right) \subset$ $C M O^{q}\left(\mathbb{Q}_{p}^{n}\right)$ and $\|b\|_{C M O q} \leq\|b\|_{C M O^{r}}$.

Proof For any $b \in C M O^{r}\left(\mathbb{Q}_{p}^{n}\right)$, by Hölder's inequality, we have

$$
\begin{aligned}
& \left(\frac{1}{\left|B_{\gamma}(0)\right|_{H}} \int_{B_{\gamma(0)}}\left|b(x)-b_{B_{\gamma(0)}}\right|^{q} d x\right)^{\frac{1}{q}} \\
& \quad \leq\left\{\frac{1}{\left|B_{\gamma}(0)\right|_{H}}\left(\int_{B_{\gamma(0)}}\left|b(x)-b_{B_{\gamma(0)}}\right|^{q \cdot \frac{r}{q}} d x\right)^{\frac{q}{r}}\left(\int_{B_{\gamma(0)}} 1 d x\right)^{1-\frac{q}{r}}\right\}^{\frac{1}{q}} \\
& \quad=\left\{\frac{1}{\left|B_{\gamma}(0)\right|_{H}}\left(\int_{B_{\gamma(0)}}\left|b(x)-b_{B_{\gamma(0)}}\right|^{r} d x\right)^{\frac{q}{r}}\left|B_{\gamma(0)}\right|_{H}^{1-\frac{q}{r}}\right\}^{\frac{1}{q}} \\
& \quad=\left(\frac{1}{\left|B_{\gamma}(0)\right|_{H}} \int_{B_{\gamma(0)}}\left|b(x)-b_{B_{\gamma(0)}}\right|^{r} d x\right)^{\frac{1}{r}} \\
& \quad \leq\|b\|_{C M O^{r}}\left(\mathbb{Q}_{p}^{n}\right) .
\end{aligned}
$$

Therefore, $b \in C M O^{q}\left(\mathbb{Q}_{p}^{n}\right)$ and $\|b\|_{C M O^{q}} \leq\|b\|_{C M O^{r}}$. This completes the proof. 
Lemma 2.2 Suppose that $b$ is a CMO function, $j, k \in \mathbb{Z}$, then

$$
\left|b(t)-b_{B_{k}}\right| \leq\left|b(t)-b_{B_{j}}\right|+p^{n}|j-k|\|b\|_{C M O^{1}\left(\mathbb{Q}_{p}^{n}\right)}
$$

Proof For $i \in \mathbb{Z}$, recall that $b_{B_{i}}=\frac{1}{\left|B_{i}\right|_{H}} \int_{B_{i}} b(x) d x$, we have

$$
\begin{aligned}
\left|b_{B_{i}}-b_{B_{i+1}}\right| & \leq \frac{1}{\left|B_{i}\right|_{H}} \int_{B_{i}}\left|b(t)-b_{B_{i+1}}\right| d t \\
& \leq \frac{p^{n}}{\left|B_{i+1}\right|_{H}} \int_{B_{i+1}}\left|b(t)-b_{B_{i+1}}\right| d t \\
& \leq p^{n}\|b\|_{C M O^{1}\left(\mathbb{Q}_{p}^{n}\right)} .
\end{aligned}
$$

For $j, k \in \mathbb{Z}$, without loss of generality, we can assume that $j \leq k$, by (2.2), we get

$$
\begin{aligned}
\left|b(t)-b_{B_{k}}\right| & \leq\left|b(t)-b_{B_{j}}\right|+\sum_{i=k}^{j-1}\left|b_{B_{i}}-b_{B_{i+1}}\right| \\
& \leq\left|b(t)-b_{B_{j}}\right|+p^{n}|j-k|\|b\|_{C M O^{1}\left(\mathbb{Q}_{p}^{n}\right)} .
\end{aligned}
$$

The lemma is proved.

Proof of Theorem 1.1 Denote $f(x) \chi_{i}(x)=f_{i}(x)$.

(1) By definition,

$$
\begin{aligned}
& \left\|\left(\mathcal{H}_{\beta, b}^{p} f\right) \chi_{k}\right\|_{L^{r_{2}\left(\mathbb{Q}_{p}^{n}\right)}}^{r_{2}}=\int_{S_{k}}|x|_{p}^{-r_{2}(n-\beta)}\left|\int_{B(0,|x| p)} f(t)(b(x)-b(t)) d t\right|^{r_{2}} d x \\
& \leq \int_{S_{k}} p^{-k r_{2}(n-\beta)}\left(\int_{B\left(0, p^{k}\right)}|f(t)(b(x)-b(t))| d t\right)^{r_{2}} d x \\
& =p^{-k r_{2}(n-\beta)} \int_{S_{k}}\left(\sum_{j=-\infty}^{k} \int_{S_{j}}|f(t)(b(x)-b(t))| d t\right)^{r_{2}} d x \\
& \leq C p^{-k r_{2}(n-\beta)} \int_{S_{k}}\left(\sum_{j=-\infty}^{k} \int_{S_{j}}\left|f(t)\left(b(x)-b_{B_{k}}\right)\right| d t\right)^{r_{2}} d x \\
& +C p^{-k r_{2}(n-\beta)} \int_{S_{k}}\left(\sum_{j=-\infty}^{k} \int_{S_{j}}\left|f(t)\left(b(t)-b_{B_{k}}\right)\right| d t\right)^{r_{2}} d x \\
& :=I+I I \text {. }
\end{aligned}
$$

Now let us estimate $I$ and $I I$, respectively. For $I$, by Hölder's inequality $\left(\frac{1}{r_{1}}+\frac{1}{r_{1}^{\prime}}=1\right)$, we have

$$
\begin{aligned}
I & =C p^{-k r_{2}(n-\beta)}\left(\int_{S_{k}}\left|b(x)-b_{B_{k}}\right|^{r_{2}} d x\right)\left(\sum_{j=-\infty}^{k} \int_{S_{j}}|f(t)| d t\right)^{r_{2}} \\
& \leq C p^{\frac{-k r_{2} n}{r_{1}^{\prime}}}\left(\frac{1}{\left|B_{k}\right|_{H}} \int_{B_{k}}\left|b(x)-b_{B_{k}}\right|^{r_{2}} d x\right)
\end{aligned}
$$




$$
\begin{gathered}
\times\left\{\sum_{j=-\infty}^{k}\left(\int_{S_{j}}|f(t)|^{r_{1}} d t\right)^{\frac{1}{r_{1}}}\left(\int_{S_{j}} d t\right)^{\frac{1}{r_{1}^{\prime}}}\right\}^{r_{2}} \\
\leq C\|b\|_{C M O^{r_{2}}\left(\mathbb{Q}_{p}^{n}\right)}^{r_{2}}\left\{\sum_{j=-\infty}^{k} p^{\frac{(j-k) n}{r_{1}^{\prime}}}\left\|f_{j}\right\|_{L^{r_{1}}\left(\mathbb{Q}_{p}^{n}\right)}\right\}^{r_{2}} .
\end{gathered}
$$

For $I I$, by Lemma 2.2, we get

$$
\begin{aligned}
I I= & C p^{-k r_{2}(n-\beta)} \int_{S_{k}}\left(\sum_{j=-\infty}^{k} \int_{S_{j}}\left|f(t)\left(b(t)-b_{B_{k}}\right)\right| d t\right)^{r_{2}} d x \\
= & C p^{-k r_{2}(n-\beta)} p^{k n}\left(1-p^{-n}\right)\left(\sum_{j=-\infty}^{k} \int_{S_{j}}\left|f(t)\left(b(t)-b_{B_{k}}\right)\right| d t\right)^{r_{2}} \\
\leq & C p^{\frac{-k r_{2} n}{r_{1}^{\prime}}}\left(\sum_{j=-\infty}^{k} \int_{S_{j}}\left|f(t)\left(b(t)-b_{B_{j}}\right)\right| d t\right)^{r_{2}} \\
& +C p^{\frac{-k r_{2} n}{r_{1}^{\prime}}}\|b\|_{C M O^{1}\left(\mathbb{Q}_{p}^{n}\right)}^{r_{2}}\left(\sum_{j=-\infty}^{k}(k-j) \int_{S_{j}}|f(t)| d t\right)^{r_{2}}=I I_{1}+I I_{2} .
\end{aligned}
$$

For $I_{1}$ and $I I_{2}$, by Hölder's inequality, we obtain

$$
\begin{aligned}
I I_{1} & \leq C p^{\frac{-k r_{2} n}{r_{1}^{\prime}}}\left\{\left(\sum_{j=-\infty}^{k} \int_{S_{j}}|f(t)|^{r_{1}} d t\right)^{\frac{1}{r_{1}}}\left(\int_{S_{j}}\left|b(t)-b_{B_{j}}\right|^{r_{1}^{\prime}} d t\right)^{\frac{1}{r_{1}^{\prime}}}\right\}^{r_{2}} \\
& \leq C p^{\frac{-k r_{2} n}{r_{1}^{\prime}}}\left\{\sum_{j=-\infty}^{k}\left\|f_{j}\right\|_{L^{\prime}\left(\mathbb{Q}_{p}^{n}\right)} p^{\frac{j n}{r_{1}^{\prime}}}\left(\frac{1}{\left|B_{j}\right|_{H}} \int_{B_{j}}\left|b(t)-b_{B_{j}}\right|^{r_{1}^{\prime}} d t\right)^{\frac{1}{r_{1}^{\prime}}}\right\}^{r_{2}} \\
& \leq C\|b\|_{\left.C M O^{\prime}{ }_{1}^{\prime} \mathbb{Q}_{p}^{n}\right)}^{r_{2}}\left\{\sum_{j=-\infty}^{k} p^{\frac{(j-k) n}{r_{1}^{\prime}}}\left\|f_{j}\right\|_{L^{r_{1}}\left(\mathbb{Q}_{p}^{n}\right)}\right\}^{r_{2}}
\end{aligned}
$$

and

$$
\begin{aligned}
I I_{2} & \leq C p^{\frac{-k r_{2} n}{r_{1}^{\prime}}}\|b\|_{C M O^{1}\left(\mathbb{Q}_{p}^{n}\right)}^{r_{2}}\left\{\sum_{j=-\infty}^{k}(k-j)\left(\int_{S_{j}}|f(t)|^{r_{1}} d t\right)^{\frac{1}{r_{1}}}\left(\int_{S_{j}} d t\right)^{\frac{1}{r_{1}^{\prime}}}\right\}^{r_{2}} \\
& \leq C\|b\|_{C M O^{1}\left(\mathbb{Q}_{p}^{n}\right)}^{r_{2}}\left\{\sum_{j=-\infty}^{k}(k-j) p^{\frac{(j-k) n}{r_{1}^{\prime}}}\left\|f_{j}\right\|_{L^{r_{1}}\left(\mathbb{Q}_{p}^{n}\right)}\right\}^{r_{2}} .
\end{aligned}
$$

Then the above inequalities together with Lemma 2.1 imply that

$$
\begin{aligned}
\left\|\mathcal{H}_{\beta, b}^{p} f\right\|_{K_{r_{2}}^{\alpha, q_{2}\left(\mathbb{Q}_{p}^{n}\right)}} & =\left(\sum_{k=-\infty}^{+\infty} p^{k \alpha q_{2}}\left\|\left(\mathcal{H}_{\beta, b}^{p} f\right) \chi_{k}\right\|_{L^{r_{2}\left(\mathbb{Q}_{p}^{n}\right)}}^{q_{2}}\right)^{\frac{1}{q_{2}}} \\
& \leq\left(\sum_{k=-\infty}^{+\infty} p^{k \alpha q_{1}}\left\|\left(\mathcal{H}_{\beta, b}^{p} f\right) \chi_{k}\right\|_{L^{r_{2}\left(\mathbb{Q}_{p}^{n}\right)}}^{q_{1}}\right)^{\frac{1}{q_{1}}}
\end{aligned}
$$




$$
\begin{aligned}
\leq & C\left\{\sum_{k=-\infty}^{+\infty} p^{k \alpha q_{1}}\|b\|_{C M O^{r_{2}}\left(\mathbb{Q}_{p}^{n}\right)}^{q_{1}}\left(\sum_{j=-\infty}^{k} p^{\frac{(j-k) n}{r_{1}^{\prime}}}\left\|f_{j}\right\|_{L^{r_{1}}\left(\mathbb{Q}_{p}^{n}\right)}\right)^{q_{1}}\right\}^{\frac{1}{q_{1}}} \\
& +C\left\{\sum_{k=-\infty}^{+\infty} p^{k \alpha q_{1}}\|b\|_{C M O^{\prime \prime}\left(\mathbb{Q}_{p}^{n}\right)}^{q_{1}}\left(\sum_{j=-\infty}^{k} p^{\frac{(j-k) n}{r_{1}^{\prime}}}\left\|f_{j}\right\|_{L^{r_{1}}\left(\mathbb{Q}_{p}^{n}\right)}\right)^{q_{1}}\right\}^{\frac{1}{q_{1}}} \\
& +C\left\{\sum_{k=-\infty}^{+\infty} p^{k \alpha q_{1}}\|b\|_{C M O^{1}\left(\mathbb{Q}_{p}^{n}\right)}^{q_{1}}\left(\sum_{j=-\infty}^{k}(k-j) p^{\frac{(j-k) n}{r_{1}^{\prime}}}\left\|f_{j}\right\|_{L^{r_{1}}\left(\mathbb{Q}_{p}^{n}\right)}\right)^{q_{1}}\right\}^{\frac{1}{q_{1}}} \\
\leq & C\|b\|_{C M O^{\left.\max r_{1}^{\prime} r_{2} r_{2}\right\}}\left(\mathbb{Q}_{p}^{n}\right)}\left\{\sum_{k=-\infty}^{+\infty} p^{k \alpha q_{1}}\left(\sum_{j=-\infty}^{k}(k-j) p^{\frac{(j-k) n}{r_{1}^{\prime}}}\left\|f_{j}\right\|_{L^{r_{1}}\left(\mathbb{Q}_{p}^{n}\right)}\right)^{q_{1}}\right\}^{\frac{1}{q_{1}}} \\
= & J .
\end{aligned}
$$

For the case $0<q_{1} \leq 1$, since $\alpha<\frac{n}{r_{1}^{\prime}}$, we have

$$
\begin{aligned}
& J^{q_{1}}=C\|b\|_{C M O^{\max \left(r_{1}^{\prime}, r_{2}\right\}}}^{q_{1}} \sum_{\left.\mathbb{Q}_{p}^{n}\right)}^{+\infty} \sum_{k=-\infty}^{k \alpha q_{1}}\left(\sum_{j=-\infty}^{k}(k-j) p^{\frac{(j-k) n}{r_{1}^{\prime}}}\left\|f_{j}\right\|_{L^{\prime}\left(\mathbb{Q}_{p}^{n}\right)}\right)^{q_{1}}
\end{aligned}
$$

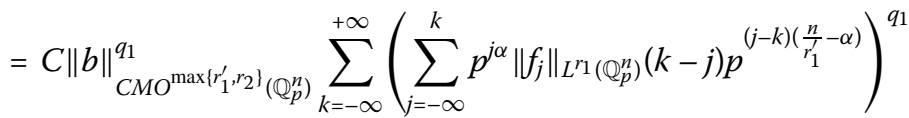

$$
\begin{aligned}
& \leq C\|b\|_{C M O^{\max \left\{r_{1}^{\prime}, r_{2}\right\}}}^{q_{1}} \sum_{\left.\mathbb{Q}_{p}^{n}\right)}^{+\infty} \sum_{k=-\infty}^{+\infty} \sum_{j=-\infty}^{k} p^{j \alpha q_{1}}\left\|f_{j}\right\|_{L^{r_{1}}\left(\mathbb{Q}_{p}^{n}\right)}^{q_{1}}(k-j)^{q_{1}} p^{(j-k)\left(\frac{n}{r_{1}^{\prime}}-\alpha\right) q_{1}} \\
& \left.=C\|b\|_{C M O^{\max }\left\{r_{1}^{\prime}, r_{2}\right\}}^{q_{(}} \sum_{p}^{n}\right) \sum_{j=-\infty}^{+\infty} p^{j \alpha q_{1}}\left\|f_{j}\right\|_{L^{r}\left(\mathbb{Q}_{p}^{n}\right)}^{q_{1}} \sum_{k=j}^{+\infty}(k-j)^{q_{1}} p^{(j-k)\left(\frac{n}{r_{1}^{\prime}}-\alpha\right) q_{1}} \\
& =C\|b\|_{\left.C M O^{\max \left\{r_{1}^{\prime}, r_{2}\right\}} \mathbb{Q}_{p}^{n}\right)}^{q_{1}}\|f\|_{K_{r_{1}}^{\alpha, r_{1}}\left(\mathbb{Q}_{p}^{n}\right)}^{q_{1}} \text {. }
\end{aligned}
$$

For the case $q_{1}>1$, by Hölder's inequality, we have

$$
\begin{aligned}
& J^{q_{1}}=C\|b\|_{C M O^{\max \left\langle r_{1}^{\prime}, r_{2}\right\}}}^{q_{1}} \sum_{\left.\mathbb{Q}_{p}^{n}\right)}^{+\infty}\left(\sum_{k=-\infty}^{k} \sum_{j=-\infty}^{j \alpha}\left\|f_{j}\right\|_{L^{r_{1}}\left(\mathbb{Q}_{p}^{n}\right)}(k-j) p^{(j-k)\left(\frac{n}{r_{1}^{\prime}}-\alpha\right)}\right)^{q_{1}} \\
& \leq C\|b\|_{\left.C M O^{\max \left\langle r_{1}^{\prime}, r_{2}\right\}} \mathfrak{Q}_{p}^{n}\right)}^{\left.q_{1}\right)} \sum_{k=-\infty}^{+\infty}\left(\sum_{j=-\infty}^{k} p^{j \alpha q_{1}}\left\|f_{j}\right\|_{L^{\prime} 1\left(\mathbb{Q}_{p}^{n}\right)}^{q_{1}} p^{\frac{(j-k)}{2}\left(\frac{n}{r_{1}^{\prime}}-\alpha\right) q_{1}}\right) \\
& \times\left(\sum_{j=-\infty}^{k}(k-j)^{q_{1}^{\prime}} p^{\frac{(j-k)}{2}\left(\frac{n}{r_{1}^{\prime}}-\alpha\right) q_{1}^{\prime}}\right)^{\frac{q_{1}}{q_{1}^{\prime}}} \\
& \left.=C\|b\|_{C M O^{\max }\left(r_{1}^{\prime}, r_{2}\right\}}^{q_{1}} \mathbb{Q}_{p}^{n}\right) \sum_{j=-\infty}^{+\infty} p^{j \alpha q_{1}}\left\|f_{j}\right\|_{L^{1}\left(\mathbb{Q}_{p}^{n}\right)}^{q_{1}} \sum_{k=j}^{+\infty} p^{\frac{(j-k)}{2}\left(\frac{n}{r_{1}^{\prime}}-\alpha\right) q_{1}} \\
& =C\|b\|_{\left.C M O^{\max \left\{r_{1}^{\prime}, r_{2}\right\}} \mathbb{Q}_{p}^{n}\right)}^{q_{1}}\|f\|_{K_{r_{1}}^{\alpha, r_{1}}\left(\mathbb{Q}_{p}^{n}\right)}^{q_{1}} .
\end{aligned}
$$

Then (1.6) follows from (2.4)-(2.6). 
(2) By definition,

$$
\begin{aligned}
& \left\|\left(\mathcal{H}_{\beta, b}^{p, *} f\right) \chi_{k}\right\|_{L^{r_{2}\left(\mathbb{Q}_{p}^{n}\right)}}^{r_{2}}=\left.\left.\int_{S_{k}}\left|\int_{\mathbb{Q}_{p}^{n} \backslash B(0,|x| p)}\right| t\right|_{p} ^{\beta-n} f(t)(b(x)-b(t)) d t\right|^{r_{2}} d x \\
& \leq \int_{S_{k}}\left(\int_{\mathbb{Q}_{p}^{n} \backslash B\left(0, p^{k}\right)}|t|_{p}^{\beta-n}|f(t)(b(x)-b(t))| d t\right)^{r_{2}} d x \\
& =\int_{S_{k}}\left(\sum_{j=k}^{\infty} \int_{S_{j}} p^{j(\beta-n)}|f(t)(b(x)-b(t))| d t\right)^{r_{2}} d x \\
& \leq C \int_{S_{k}}\left(\sum_{j=k}^{\infty} \int_{S_{j}} p^{j(\beta-n)}\left|f(t)\left(b(x)-b_{B_{k}}\right)\right| d t\right)^{r_{2}} d x \\
& +C \int_{S_{k}}\left(\sum_{j=k}^{\infty} \int_{S_{j}} p^{j(\beta-n)}\left|f(t)\left(b(t)-b_{B_{k}}\right)\right| d t\right)^{r_{2}} d x \\
& :=K+L \text {. }
\end{aligned}
$$

By Hölder's inequality, we get

$$
\begin{aligned}
K= & C\left(\int_{S_{k}}\left|b(x)-b_{B_{k}}\right|^{r_{2}} d x\right)\left(\sum_{j=k}^{\infty} \int_{S_{j}} p^{j(\beta-n)}|f(t)| d t\right)^{r_{2}} \\
\leq & C p^{k n}\left(\frac{1}{\left|B_{k}\right|_{H}} \int_{B_{k}}\left|b(x)-b_{B_{k}}\right|^{r_{2}} d x\right) \\
& \times\left\{\sum_{j=k}^{\infty}\left(\int_{S_{j}}|f(t)|^{r_{1}} d t\right)^{\frac{1}{r_{1}}} p^{j\left(\beta-\frac{n}{r_{1}}\right)}\right\}^{r_{2}} \\
\leq & C\|b\|_{C M O^{r_{2}\left(\mathbb{Q}_{p}^{n}\right)}}^{r_{2}}\left\{\sum_{j=k}^{\infty} p^{\frac{(k-j) n}{r_{2}}}\left\|f_{j}\right\|_{L^{r_{1}}\left(\mathbb{Q}_{p}^{n}\right)}\right\}^{r_{2}} .
\end{aligned}
$$

By Lemma 2.2, we have

$$
\begin{aligned}
L= & C \int_{S_{k}}\left(\sum_{j=k}^{\infty} \int_{S_{j}} p^{j(\beta-n)}\left|f(t)\left(b(t)-b_{B_{k}}\right)\right| d t\right)^{r_{2}} d x \\
= & C p^{k n}\left(1-p^{-n}\right)\left(\sum_{j=k}^{\infty} \int_{S_{j}} p^{j(\beta-n)}\left|f(t)\left(b(t)-b_{B_{k}}\right)\right| d t\right)^{r_{2}} \\
\leq & C p^{k n}\left(\sum_{j=k}^{\infty} \int_{S_{j}} p^{j(\beta-n)}\left|f(t)\left(b(t)-b_{B_{j}}\right)\right| d t\right)^{r_{2}} \\
& +C p^{k n}\|b\|_{C M O^{1}\left(\mathbb{Q}_{p}^{n}\right)}^{r_{2}}\left(\sum_{j=k}^{\infty}(j-k) p^{j(\beta-n)} \int_{S_{j}}|f(t)| d t\right)^{r_{2}} \\
= & L_{1}+L_{2} .
\end{aligned}
$$


For $L_{1}$ and $L_{2}$, by Hölder's inequality, we obtain

$$
\begin{aligned}
L_{1} & \leq C p^{k n}\left\{\sum_{j=k}^{\infty} p^{j(\beta-n)}\left(\int_{S_{j}}\left|b(t)-b_{B_{j}}\right|^{r_{1}^{\prime}} d t\right)^{\frac{1}{r_{1}^{\prime}}}\left(\int_{S_{j}}|f(t)|^{r_{1}} d t\right)^{\frac{1}{r_{1}}}\right\}^{r_{2}} \\
& \leq C p^{k n}\left\{\sum_{j=k}^{\infty} p^{\frac{j n}{r_{1}^{\prime}}+j(\beta-n)}\left\|f_{j}\right\|_{L^{r_{1}}\left(\mathbb{Q}_{p}^{n}\right)}\left(\frac{1}{\left|B_{j}\right|_{H}} \int_{B_{j}}\left|b(t)-b_{B_{j}}\right|^{r_{1}^{\prime}} d t\right)^{\frac{1}{r_{1}^{\prime}}}\right\}^{r_{2}} \\
& \leq C\|b\|_{C M O^{r_{1}^{\prime}}\left(\mathbb{Q}_{p}^{n}\right)}^{r_{2}}\left\{\sum_{j=k}^{\infty} p^{\frac{(k-j) n}{r_{2}}}\left\|f_{j}\right\|_{L^{r_{1}\left(\mathbb{Q}_{p}^{n}\right)}}\right\}^{r_{2}}
\end{aligned}
$$

and

$$
\begin{aligned}
L_{2} & \leq C\|b\|_{C M O^{1}\left(\mathbb{Q}_{p}^{n}\right)}^{r_{2}} p^{k n}\left\{\sum_{j=k}^{\infty}(j-k) p^{j(\beta-n)}\left(\int_{S_{j}}|f(t)|^{r_{1}} d t\right)^{\frac{1}{r_{1}}}\left(\int_{S_{j}} d t\right)^{\frac{1}{r_{1}^{\prime}}}\right\}^{r_{2}} \\
& \leq C\|b\|_{C M O^{1}\left(\mathbb{Q}_{p}^{n}\right)}^{r_{2}}\left\{\sum_{j=k}^{\infty}(j-k) p^{\frac{(k-j) n}{r_{2}}}\left\|f_{j}\right\|_{L^{r_{1}\left(\mathbb{Q}_{p}^{n}\right)}}\right\}^{r_{2}} .
\end{aligned}
$$

Then (2.7)-(2.8) together with Lemma 2.1 imply that

$$
\begin{aligned}
& \left\|\mathcal{H}_{\beta, b}^{p, *} f\right\|_{K_{r_{2}}^{\alpha, q_{2}}\left(\mathbb{Q}_{p}^{n}\right)}=\left(\sum_{k=-\infty}^{+\infty} p^{k \alpha q_{2}}\left\|\left(\mathcal{H}_{\beta, b}^{p, *} f\right) \chi_{k}\right\|_{L^{r_{2}\left(\mathbb{Q}_{p}^{n}\right)}}^{q_{2}}\right)^{\frac{1}{q_{2}}} \\
& \leq\left(\sum_{k=-\infty}^{+\infty} p^{k \alpha q_{1}}\left\|\left(\mathcal{H}_{\beta, b}^{p, *} f\right) \chi_{k}\right\|_{L^{r_{2}\left(\mathbb{Q}_{p}^{n}\right)}}^{q_{1}}\right)^{\frac{1}{q_{1}}} \\
& \leq C\left(\sum_{k=-\infty}^{+\infty} p^{k \alpha q_{1}}\|b\|_{C M O^{2}\left(\mathbb{Q}_{p}^{n}\right)}^{q_{1}}\left(\sum_{j=k}^{\infty} p^{\frac{(k-j) n}{r_{2}}}\left\|f_{j}\right\|_{L^{r_{1}}\left(\mathbb{Q}_{p}^{n}\right)}\right)^{q_{1}}\right)^{\frac{1}{q_{1}}} \\
& +C\left(\sum_{k=-\infty}^{+\infty} p^{k \alpha q_{1}}\|b\|_{C M O^{r_{1}^{\prime}\left(\mathbb{Q}_{p}^{n}\right)}}^{q_{1}}\left(\sum_{j=k}^{\infty} p^{\frac{(k-j) n}{r_{2}}}\left\|f_{j}\right\|_{L^{r_{1}\left(\mathbb{Q}_{p}^{n}\right)}}\right)^{q_{1}}\right)^{\frac{1}{q_{1}}} \\
& +C\left(\sum_{k=-\infty}^{+\infty} p^{k \alpha q_{1}}\|b\|_{C M O^{1}\left(\mathbb{Q}_{p}^{n}\right)}^{q_{1}}\left(\sum_{j=k}^{\infty}(k-j) p^{\frac{(j-k) n}{r_{2}}}\left\|f_{j}\right\|_{L^{r_{1}}\left(\mathbb{Q}_{p}^{n}\right)}\right)^{q_{1}}\right)^{\frac{1}{q_{1}}}
\end{aligned}
$$

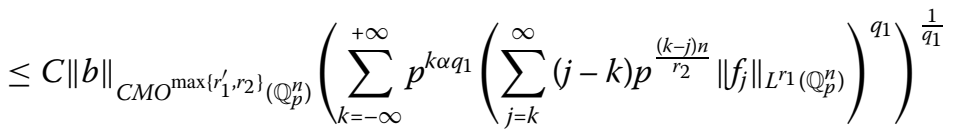

$$
\begin{aligned}
& =S \text {. }
\end{aligned}
$$

For the case $0<q_{1} \leq 1$, since $\alpha>-\frac{n}{r_{2}}$, we have

$$
\begin{aligned}
S^{q_{1}} & =C\|b\|_{C M O^{\max \left\{r_{1}^{\prime}, r_{2}\right\}}}^{q_{1}} \sum_{\left.\mathbb{Q}_{p}^{n}\right)}^{+\infty} \sum_{k=-\infty}^{+\infty \alpha q_{1}}\left(\sum_{j=k}^{\infty}(j-k) p^{\frac{(k-j) n}{r_{2}}}\left\|f_{j}\right\|_{L^{r_{1}}\left(\mathbb{Q}_{p}^{n}\right)}\right)^{q_{1}} \\
& =C\|b\|_{C M O^{\max \left\{r_{1}^{\prime}, r_{2}\right\}}}^{q_{1}} \sum_{\left.\mathbb{Q}_{p}^{n}\right)}^{+\infty}\left(\sum_{k=-\infty}^{\infty} p_{j=k}^{j \alpha}\left\|f_{j}\right\|_{L^{r_{1}}\left(\mathbb{Q}_{p}^{n}\right)}(j-k) p^{(k-j)\left(\frac{n}{r_{2}}+\alpha\right)}\right)^{q_{1}}
\end{aligned}
$$




$$
\begin{aligned}
& \leq C\|b\|_{\left.C M O^{\max \left\{r_{1}^{\prime}, r_{2}\right\}} \mathbb{Q}_{p}^{n}\right)}^{q_{1}} \sum_{k=-\infty}^{+\infty} \sum_{j=k}^{\infty} p^{j \alpha q_{1}}\left\|f_{j}\right\|_{L^{r_{1}}\left(\mathbb{Q}_{p}^{n}\right)}^{q_{1}}(j-k)^{q_{1}} p^{(k-j)\left(\frac{n}{r_{2}}+\alpha\right) q_{1}} \\
& =C\|b\|_{C M O^{\max \left\{r_{1}^{\prime}, r_{2}\right\}}{ }_{\left(\mathbb{Q}_{p}^{n}\right)}^{q_{1}}} \sum_{j=-\infty}^{+\infty} p^{j \alpha q_{1}}\left\|f_{j}\right\|_{L^{r_{1}}\left(\mathbb{Q}_{p}^{n}\right)}^{q_{1}} \sum_{k=-\infty}^{j}(j-k)^{q_{1}} p^{(k-j)\left(\frac{n}{r_{2}}+\alpha\right) q_{1}} \\
& =C\|b\|_{\left.C M O^{\max \left\{r_{1}^{\prime}, r_{2}\right\}} \mathbb{Q}_{p}^{n}\right)}^{\left.q_{1}\right)}\|f\|_{K_{r_{1}}^{\alpha, q_{1}}\left(\mathbb{Q}_{p}^{n}\right)}^{q_{1}} .
\end{aligned}
$$

For the case $q_{1}>1$, by Hölder's inequality, we have

$$
\begin{aligned}
& S^{q_{1}}=C\|b\|_{C M O^{\max }\left(r_{1}^{\prime}, r_{2}\right\}}^{\left.q_{(\mathbb{Q}}\right)} \sum_{k=-\infty}^{+\infty} p^{k \alpha q_{1}}\left(\sum_{j=k}^{\infty}(j-k) p^{\frac{(k-j) n}{r_{2}}}\left\|f_{j}\right\|_{L^{r_{1}}\left(\mathbb{Q}_{p}^{n}\right)}\right)^{q_{1}} \\
& \leq C\|b\|_{C M O^{\max }\left\langle r_{1}^{\prime}, r_{2}\right\}}^{\left.q_{(} \mathbb{Q}_{p}^{n}\right)} \sum_{k=-\infty}^{+\infty}\left(\sum_{j=k}^{\infty} p^{j \alpha q_{1}}\left\|f_{j}\right\|_{L^{r_{1}}\left(\mathbb{Q}_{p}^{n}\right)}^{q_{1}} p^{\frac{(k-j)}{2}\left(\frac{n}{r_{2}}+\alpha\right) q_{1}}\right) \\
& \times\left(\sum_{j=k}^{\infty}(j-k)^{q_{1}^{\prime}} p^{\frac{(k-j)}{2}\left(\frac{n}{r_{2}}+\alpha\right) q_{1}^{\prime}}\right)^{\frac{q_{1}}{q_{1}^{\prime}}} \\
& =C\|b\|_{C M O^{\max \left\langle r_{1}^{\prime}, r_{2}\right\}}}^{q_{1}} \sum_{\left.\mathbb{Q}_{p}^{n}\right)}^{+\infty} \sum_{j=-\infty}^{j \alpha q_{1}}\left\|f_{j}\right\|_{L^{r_{1}}\left(\mathbb{Q}_{p}^{n}\right)}^{q_{1}} \sum_{k=-\infty}^{j} p^{\frac{(k-j)}{2}\left(\frac{n}{r_{2}}+\alpha\right) q_{1}} \\
& =C\|b\|_{C M O^{\left.\max r_{1}^{\prime}, r_{2}\right\}}{ }_{\left(\mathbb{Q}_{p}^{n}\right)}^{\left.q_{1}\right)}}^{q_{1}}\|f\|_{K_{r_{1}}^{\alpha, q_{1}}\left(\mathbb{Q}_{p}^{n}\right)}^{q_{1}} .
\end{aligned}
$$

Then the above inequalities imply (1.7). Theorem 1.1 is proved.

\section{Competing interests}

The author declares that she has no competing interests.

\section{Acknowledgements}

The author sincerely thanks Professor Zunwei Fu for his useful discussions. This work was supported by NSF of China (Grant No. 11126203), NSF of Shandong Province (Grant Nos. ZR2010AL006).

\section{Received: 20 July 2012 Accepted: 23 November 2012 Published: 11 December 2012}

\section{References}

1. Albeverio, S, Karwoski, W: A random walk on p-adics: the generator and its spectrum. Stoch. Process. Appl. 53, 1-22 (1994)

2. Avetisov, AV, Bikulov, AH, Kozyrev, SV, Osipov, VA: -adic models of ultrametric diffusion constrained by hierarchical energy landscapes. J. Phys. A, Math. Gen. 35, 177-189 (2002)

3. Khrennikov, A: p-Adic Valued Distributions in Mathematical Physics. Kluwer, Dordrecht (1994)

4. Khrennikov, A: Non-Archimedean Analysis: Quantum Paradoxes, Dynamical Systems and Biological Models. Kluwer, Dordrecht (1997)

5. Varadarajan, VS: Path integrals for a class of $p$-adic Schrödinger equations. Lett. Math. Phys. 39, 97-106 (1997)

6. Vladimirov, VS, Volovich, IV: $p$-adic quantum mechanics. Commun. Math. Phys. 123, 659-676 (1989)

7. Vladimirov, VS, Volovich, IV, Zelenov, El: $p$-Adic Analysis and Mathematical Physics. Series on Soviet and East European Mathematics, vol. I. World Scientific, Singapore (1992)

8. Volovich, IV: $p$-adic space-time and the string theory. Theor. Math. Phys. 71, 337-340 (1987)

9. Volovich, IV: $p$-adic string. Class. Quantum Gravity 4, 83-87 (1987)

10. Kim, YC: Carleson measures and the BMO space on the $p$-adic vector space. Math. Nachr. 282, 1278-1304 (2009)

11. Kim, YC: Weak type estimates of square functions associated with quasiradial Bochner-Riesz means on certain Hardy spaces. J. Math. Anal. Appl. 339, 266-280 (2008)

12. Rim, KS, Lee, J: Estimates of weighted Hardy-Littlewood averages on the $p$-adic vector space. J. Math. Anal. Appl. 324, 1470-1477 (2006)

13. Rogers, KM: A van der Corput lemma for the $p$-adic numbers. Proc. Am. Math. Soc. 133, 3525-3534 (2005)

14. Rogers, KM: Maximal averages along curves over the $p$-adic numbers. Bull. Aust. Math. Soc. 70, 357-375 (2004)

15. Taibleson, MH: Fourier Analysis on Local Fields. Princeton University Press, Princeton (1975) 
16. Hardy, GH: Note on a theorem of Hilbert. Math. Z. 6, 314-317 (1920)

17. Haran, S: Riesz potentials and explicit sums in arithmetic. Invent. Math. 101, 697-703 (1990)

18. Faris, W: Weak Lebesgue spaces and quantum mechanical binding. Duke Math. J. 43, 365-373 (1976)

19. Fu, ZW, Grafakos, L, Lu, SZ, Zhao, FY: Sharp bounds for m-linear Hardy and Hilbert operators. Houst. J. Math. 38 225-244 (2012)

20. Long, SC, Wang, J: Commutators of Hardy operators. J. Math. Anal. Appl. 274, 626-644 (2002)

21. Fu, ZW, Wu, QY, Lu, SZ: Sharp estimates for $p$-adic Hardy, Hardy-Littlewood-Polya operators and commutators. Acta Math. Sin. (Engl. Ser.). Preprint

22. Fu, ZW, Liu, ZG, Lu, SZ, Wang, HB: Characterization for commutators of $n$-dimensional fractional Hardy operators. Sci. China Ser. A 50, 1418-1426 (2007)

23. Fu, ZW, Lu, SZ: Commutators of generalized Hardy operators. Math. Nachr. 282, 832-845 (2009)

24. Chen, YZ, Lau, KS: Some new classes of Hardy spaces. J. Funct. Anal. 84, 255-278 (1989)

25. Garcia-Cuerva, J: Hardy spaces and Beurling algebras. J. Lond. Math. Soc. 39, 499-513 (1989)

26. Lu, SZ, Yang, DC: The central BMO spaces and Littlewood-Paley operators. Approx. Theory Appl. 11, $72-94$ (1995)

27. Zhu, YP, Zheng, WX: Besov spaces and Herz spaces on local fields. Sci. China Ser. A 41, 1051-1060 (1998)

doi:10.1186/1029-242X-2012-293

Cite this article as: Wu: Boundedness for commutators of fractional p-adic Hardy operators. Journal of Inequalities and Applications 2012 2012:293.

\section{Submit your manuscript to a SpringerOpen ${ }^{\circ}$ journal and benefit from:}

- Convenient online submission

- Rigorous peer review

Immediate publication on acceptance

Open access: articles freely available online

- High visibility within the field

- Retaining the copyright to your article 